\title{
Possible Role of Sphingolipids in Developmental Programmed Cell Death of Petunia Petals
}

\author{
Domenico Prisa ${ }^{* 1}$, Gianluca Burchi ${ }^{2}$ and Wouter G Van Doorn ${ }^{2}$ \\ ${ }^{1}$ Research Unit for the Nursery and the Management of Environmental and Ornamental Green, Pescia (PT), Italy \\ ${ }^{2}$ Mann Laboratory, Department of Plant Sciences, University of California, Davis, CA 95616, USA
}

*Corresponding author: Prisa D, Consiglio per la Ricerca e la Sperimentazione in Agricoltura (CREA-VIV), Via dei Fiori 8, 51012 Pescia, Italy Tel: 0039 3391062935; Email: domenico.prisa@gmail.com

Rec date: Oct 13, 2015, Acc date: Nov 25, 2015, Pub date: Dec 03, 2015

Copyright: @ 2015 Prisa D, et al. This is an open-access article distributed under the terms of the Creative Commons Attribution License, which permits unrestricted use, distribution, and reproduction in any medium, provided the original author and source are credited.

\begin{abstract}
A type of programmed cell death (PCD) in animals is due to lysosomal membrane permeabilisation or rupture. One of the suggested reasons is sphingolipid accumulation in the membrane. In plants many examples of PCD, including petal senescence, are due to vacuolar permeabilisation and rupture. Using Petunia flowers, we tested the effect on PCD of sphingolipids and of sphinglolipid synthesis inhibitors. Feeding flowers with five main sphingolipids (sphinganine, phytosphingosine, dihydroceramide, ceramide and sphingosine) hastened PCD. Feeding phytosphingosine together with silver thiosulphate, an inhibitor of ethylene perception, completely prevented the PCD-promoting effect of phytosphingosine, suggesting that its acts through ethylene. Feeding together with the reactive oxygen species scavenger $\mathrm{N}$-acetylcysteine had no effect, while the scavenger ascorbic acid only had a slight alleviating effect. These data suggested that phytosphingosine did not act through reactive oxygen species. Treatments with four inhibitors of sphingolipid synthesis (desipramide, fumonisin B1, myriocin, N,Ndimethylsphingosine) hastened PCD. The data indicate that changes in the endogenous sphingolipid levels resulted in early PCD. The effect of phytosphingosine was abrogated by blocking ethylene perception, suggesting that it acted upstream of ethylene, thus not by directly affecting vacuolar membrane composition.
\end{abstract}

Keywords: Petunia; Ceramide; Desipramine; Dihydroceramide; Fumonisin B1; Myriocin, N,N-Dimethylsphingosine; Petal; Phytosphingosine; Programmed cell death; Sphinganine, Sphingolipids; Sphingosine; Vacuolar membrane

\section{Introduction}

Programmed cell death (PCD) during normal plant development and during stress, i.e. plant PCD that is not due to pathogens, is generally due to permeabilisation or rupture of the vacuolar membrane. This results in the release of many hydrolases from the vacuole. These hydrolases destroy the cytoplasm and thereby kill the cell [1-3]. It is still completely unclear what the mechanical cause is of the permeabilisation or rupture of the vacuolar membrane, and how the process is activated.

One type of PCD in animal cells is very similar to the PCD during normal plant development. In this type death is also due to loss of semipermeability of the membrane of a lytic compartment (the lysosome). As in plants, this loss results in the release of molecules from the lysosome to the cytoplasm. This in turn causes cell death $[4,5]$.

One of the causes of lysosomal membrane permeabilisation in animal cells, it has been suggested, is sphingolipid accumulation. Sphingolipids are molecules containing a fatty acid and a long chain aliphatic amino alcohol. Sphingolipids, if present at low concentrations, are essential components of many membranes, including the plasma membrane, of all eukaryotic cells. If it accumulates in membranes, however, sphingosine disrupts the membranes. The detergent properties of sphingosine seem the cause of the permeabilisation of the lysosomal membrane. In animal systems sphingosine derives from ceramide, hence its synthesis is catalysed by ceramidase. Blocking ceramidase activity abrogated lysosomal permeabilisation and animal cell death [6]. Ceramide is another sphingolipid with detergent properties, which has been implicated in lysosomal permeabilisation and PCD [7]. In animal cells it can form pores (channels) in membranes. Treatment of plant cells with ceramide induced PCD, but accumulation in the membrane was apparently not required [8]. Sphingolipids such as ceramide are also secondary messengers in animal cells, thus their effect on PCD might be more indirect than through accumulation in the lysosomal membrane [7,9].

In plants much less is known about the possible role of sphingolipids in PCD [10]. Feeding with sphingolipids induced PCD in plants $[11-13,8]$. Two intermediate reactions have been proposed: an increase in the levels of calcium ions in the cytosol and an increase in reactive oxygen species (ROS). Data reviewed by Thuleau et al. in 2013 suggested a role of calcium in the induction of plant PCD by sphingolipids. Downregulation of the enzyme serine palmitoyltransferase (SPT), which catalyses the initial step in sphingolipid synthesis, prevented the increase in ROS and also prevented PCD [8].

Some examples of PCD induced by plant pathogens were found to correlate with changes in sphingolipid levels. Fumonisin, a toxin secreted by pathogenic fungi, induces PCD in the plant host. Fumonisin disrupts sphingolipid biosynthesis by blocking sphinganine $\mathrm{N}$-acyltransferase. This inhibition has been suggested to result in lethal accumulation of sphingolipid intermediates $[14,15]$.

As in animals, plant sphingolipids have two carbon chains, an aliphatic amino alcohol chain and a fatty acid chain. The major plant 
sphingolipids typically contain a C18 aliphatic amino alcohol chain (also called sphingoid long-chain base). The aliphatic amino alcohol chain can be dihydroxylated (d) or trihydroxylated $(t)$ [16,17]. Plant sphingolipids have a fatty acid chain of various lengths (about C14 to C28), but the preponderance of plant sphingolipids has a C16 fatty acid chain $[18,19]$.

Petal wilting in Petunia flowers is an example of developmental PCD that is regulated by ethylene [20]. It is likely due to vacuolar membrane permeabilization, as in many other examples of developmental PCD [21]. We here tested the idea that sphingolipids are involved in PCD. We fed the flowers with various sphingolipids. As PCD in Petunia flowers is regulated by ethylene [22], we also fed plants with sphingolipids together with silver thiosulphate, an inhibitor of ethylene perception, to determine if the sphingolipids affect PCD through ethylene. Additionally, we fed plants with sphingolipids together with $\mathrm{N}$-acetyl-L-cyteine and with ascorbic acid, both scavengers of reactive oxygen species (ROS), in order to find evidence for the possible role of ROS in inducing PCD. We also tested the effect of five inhibitors of sphingolipid biosynthesis, in an attempt to determine if the accumulation of some endogenous sphingolipids would be more important than that of others.

\section{Materials and Methods}

\section{Plant material}

Potted Petunia x hybrida cv. Baby Pink Morn (Surfinia group) plants, growing in a peat soil, were obtained from a local grower and were further grown in the laboratory greenhouse. Plants were watered automatically by misting from above. Experiments were carried out in spring and early summer with greenhouse temperatures varying between about $8^{\circ}$ (minimum at night) and $23^{\circ} \mathrm{C}$ (maximum during the daytime). One day prior to an experiment the open flowers were removed. On the day of the experiment the newly opened (fully open) flowers were pinched off by hand, and brought dry to the laboratory, within $10 \mathrm{~min}$. A small part of the end of the pedicel was removed in air, with about $5 \mathrm{~mm}$ of pedicel remaining. The flowers were immediately individually placed in small plastic containers with aqueous solutions.

\section{Time to visible PCD}

Isolated flowers were held in a controlled room at $20^{\circ} \mathrm{C}$, about $60 \%$ $\mathrm{RH}$, and a photon flux density of about $10 \mu \mathrm{mol} \mathrm{m}-2 \mathrm{~s}-1$ from Philips TDL $36 \mathrm{~W} / 84 \mathrm{cool}$ white fluorescent tubes. The lights were on for 12 continuous h per day. Visible petal wilting was used as an indicator of PCD. PCD in the petals was also accompanied by petal bluing, which was visible about one day prior to petal wilting. The colour change is likely due to an increase in vacuolar $\mathrm{pH}$ in the vacuole, which changes the colour of the anthocyanins $[23,24]$. Anthocyanins in Petunia petals are localised in the vacuole of the epidermal cells [25]. This bluing was a good indicator of impending wilting.

\section{Chemicals}

Sphingosine (d18:1, D-erythro-sphingosine) was obtained from Matreya (Pleasant Gap, PA). The other sphingolipids were purchased from Avanti Polar Lipids (Birmingham, AL): sphinganine (d18:0, Derythro-dihydrosphingosine), phytosphingosine (t18:0, 4-hydroxysphinganine), C2 dihydroceramide, and C2 ceramide. The sphingolipids were dissolved in $95 \%$ ethanol. A dilution series was made, whereby the ethanol was also serially diluted. Controls for the ethanol concentrations were included in the tests.

Inhibitors were obtained from Sigma Aldrich (Milan, Italy) unless indicated otherwise. N,N-dimethylsphingosine (DMS) was bought from from Calbiochem (Merck, Darmstadt, Germany) and fumonisin B1 from Tocris (Bristol, UK). Chemicals were directly dissolved in water except myriocin and DMS which were first dissolved in $5 \mathrm{ml}$ pure DMSO. Silver thiosulphate was made by adding a solution of silver nitrate to one of 8 times excess sodium thiosulphate.

\section{Results}

\section{PCD and symptoms observed with the naked eye}

In Petunia petals some cells in the mesophyll started to die before the onset of visible bluing and petal wilting, which are the PCD symptoms that are visible with the naked eye. Holes were formed in the mesophyll due to the local disappearance of mesophyll cells as a result of death. Death was associated with complete degradation of the cytoplasm and most of the cell walls. Only by the time the epidermis cells lost turgor, wilting (i.e. turgor loss) in the entire petal was visible with the naked eye (data not shown).

\section{Feeding with intermediates of sphingolipid biosynthesis}

Figure 1 gives a scheme of sphingolipid biosynthesis in plants, according to Berkey et al. (2012). The pathway seems different in animals, where dihydrosphingosine is converted by ceramide synthase to dihydroxyceramide, which can be desaturated to form ceramide. Ceramidases can degrade ceramide to sphingosine [26].

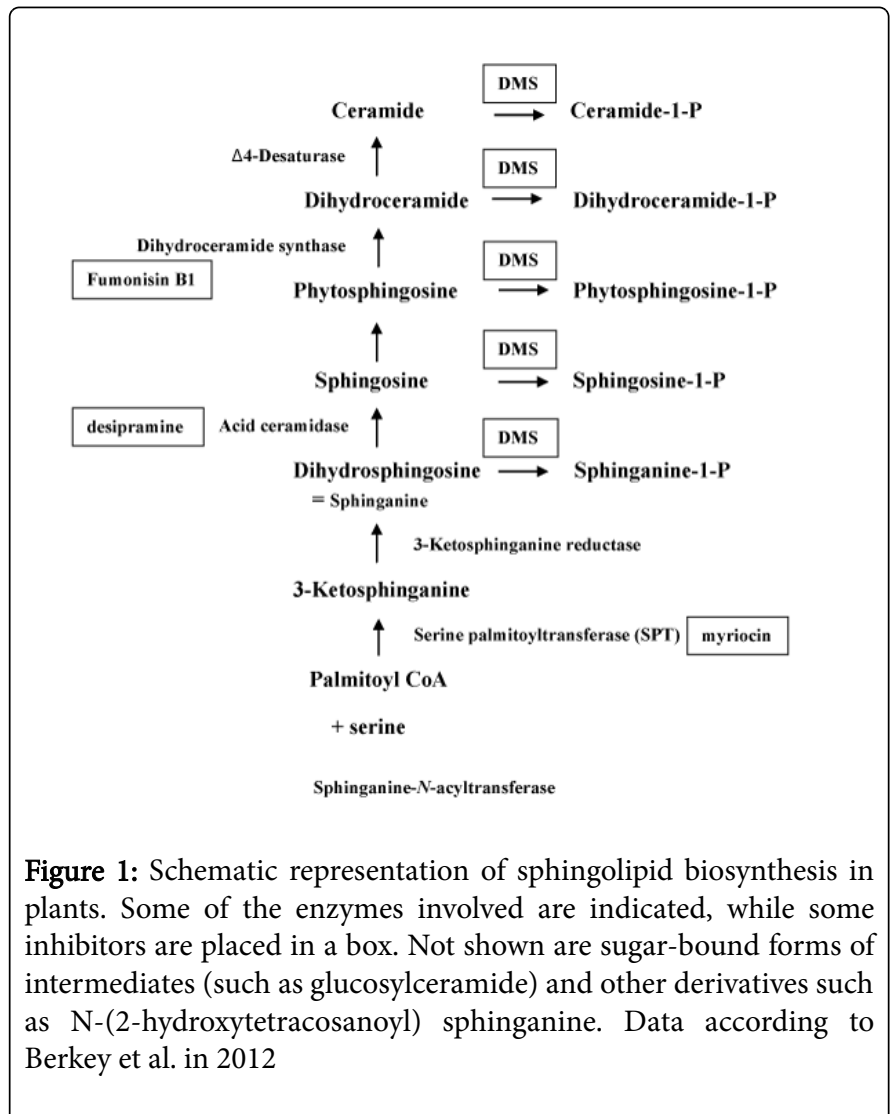


The main intermediates in the pathway (sphinganine, phytosphingosine, dihydroceramide, ceramide and sphingosine) were applied to cut flowers. Each of these resulted in a concentrationdependent decrease in the time to PCD (Figure 2). Ethanol, used as a solvent, also had a small effect, but its effect was independent of the concentration used (2-70 mM; Figure 2). These data suggest that the cells were sensitive to an increase in endogenous sphingolipid levels. The data do not show how the exogenous sphingolipids exerted their effects. Possibilities are: insertion into the plasma membrane and/or internal membranes, an increase in active oxygen species, and stimulation of effects of endogenous ethylene.

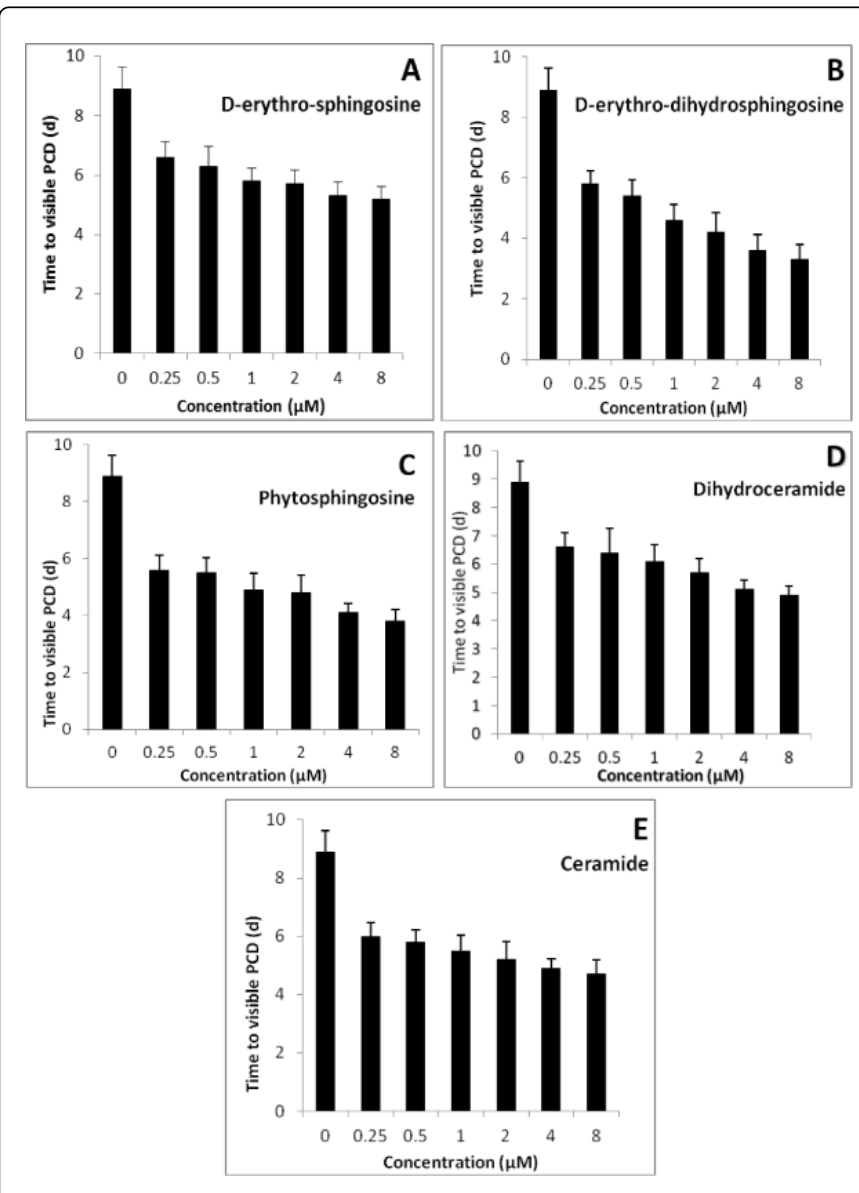

Figure 2: Effect of continuous treatment with sphingolipids on the time to visible PCD in petals of isolated Petunia flowers placed with the pedicel in aqueous solution. The external PCD symptom was wilting. Sphingolipids were all dissolved in ethanol before placement in water. (A) sphingosine (d18:1, D-erythrosphingosine), (B) dihydrosphingosine (d18:0, D-erythrodihydrosphingosine; sphinganine), (C) phytosphingosine (t18:0, 4hydroxysphinganine), (D) C2 dihydroceramide (C2 D-erythrodihydroceramide), (E) C2 ceramide (d18:1/2:0, D-erythro-Nacetylsphinganine).

\section{Phytosphingosine and silver thiosulphate (STS)}

Phytospingosine by itself, applied continuously at $1 \mu \mathrm{M}$, decreased the time to visible PCD from 8.0 to 4.9 days. Continuous feeding with $0.5,1.0$ or $2.0 \mathrm{mM}$ STS increased the time to visible PCD from 8 days to $9.3,10.1$ and 11.0 days, respectively. Differences were statistically significant with the exception of that between 9.3 and 10.1 days (Figure $3 \mathrm{~A})$. The further inclusion of $1 \mu \mathrm{M}$ phytosphingosine had no effect on the effect of STS, except when applied in combination with $2.0 \mathrm{mM}$ STS, where it slightly reduced the time to visible wilting (Figure 3A). The data show that STS completely alleviated the negative effect of phytosphingosine on the time to visible PCD, and that phytosphingosine was only slightly able to reduce the effect of STS.

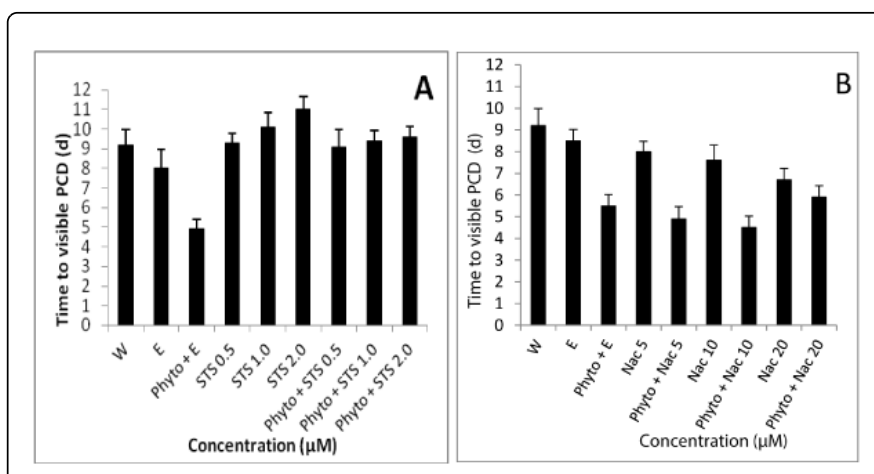

Figure 3: Combined effect of continuous treatment with phytosphingosine (t18:0, 4-hydroxy-sphinganine) with silver thiosulphate (STS) or with N-acetyl-L-cysteine (Nac), on the time to visible PCD in petals of isolated Petunia flowers placed with the pedicel in aqueous solution. The external PCD symptom was wilting. (A) STS, (B) Nac.

\section{Phytosphingosine and N-acetyl-L-cysteine (Nac)}

Two experiments were carried out. In the first Nac was fed continuously at 20,40 and $80 \mathrm{mM}$. This hastened the time to visible PCD (Figure 3B). At $20 \mathrm{mM}, \mathrm{Nac}$ by itself resulted in a time to visible PCD of 6.7 days, about $1.5 \mathrm{~d}$ less than that in the controls (Figure $3 \mathrm{~B}$ ). As indicated in the previous paragraph phytospingosine at $1 \mu \mathrm{M}$ by itself decreased the time to visible PCD to 4.9 days. The combination of $20 \mathrm{mM} \mathrm{Nac}$ and phytospingosine at $1 \mu \mathrm{M}$ resulted in a time to visible PCD of 5.9 days. The difference with the effect of phytospingosine alone was not significant (Figure 3B). The data of the higher doses of $\mathrm{Nac}$ in this experiment could not be used as their effect was close to that of phytosphingosine (data not shown). The second experiment used 5 and $10 \mathrm{mM} \mathrm{Nac}$. At $5 \mathrm{~mm} \mathrm{Nac}$ had no effect on PCD when compared to the ethanol control, while at $10 \mathrm{mM}$ it slightly hastened PCD. In this experiment phytospingosine at $1 \mu \mathrm{M}$ produced PCD after 5.5 days. The combination of this phytospingosine concentration and either concentration of $\mathrm{Nac}$ did not alleviate the effects of the phytosphingosin (Figure 3B).

\section{Phytosphingosine and ascorbid acid}

Ascorbic acid was applied at 1,5 and $10 \mathrm{mM}$. It slightly delayed the time to senescence, at 5 and $10 \mathrm{Mm}$ (Figure 3B). Ascorbid acid also slightly alleviated the negative effect of phytosphingosine on the time to senescence. The effect was absent at $1 \mathrm{mM}$, very small at $5 \mathrm{mM}$ and a bit larger at $10 \mathrm{mM}$ (Figure $3 \mathrm{~B}$ ). At the concentrations tested the effect of ascorbic acid on the control flowers was similar to the effect together with phytosphingosine (Figure 3B). 


\section{Phytosphingosine and lanthanum}

Lanthanum ions hastened PCD, from the lowest concentration tested $(0.25 \mu \mathrm{M}$; Table 1$)$. Higher concentrations, up to $8.0 \mu \mathrm{M}$, hastened PCD increasingly more (data not shown). Phytosphingo-sine was tested at $8.0 \mu \mathrm{M}$, together with $0.25 \mu \mathrm{M}$ or $0.50 \mathrm{mM} \mathrm{LaCl} 3$. The lanthanum treatments did not alleviate the effect of phytosphingosine (Table 1).

\begin{tabular}{|l|l|}
\hline Time to visible wilting (d)1 & \\
\hline Control (distilled water) & $8.9 \pm 0.3 \mathrm{a}$ \\
\hline Ethanol $70 \mathrm{mM}$ & $7.9 \pm 0.4 \mathrm{~b}$ \\
\hline LaCl3 $0.25 \mu \mathrm{M}$ & $5.4 \pm 0.5 \mathrm{c}$ \\
\hline LaCl3 $0.50 \mu \mathrm{M}$ & $5.3 \pm 0.4 \mathrm{c}$ \\
\hline Phytosphingosine 8 $\mathrm{MM}$ & $5.9 \pm 0.4 \mathrm{c}$ \\
\hline Phytosphingosine 8 $\mu \mathrm{M}+\mathrm{LaCl} 30.25 \mu \mathrm{M}$ & $5.8 \pm 0.3 \mathrm{c}$ \\
\hline Phytosphingosine 8 $\mu \mathrm{M}+\mathrm{LaCl} 30.50 \mu \mathrm{M}$ & $5.6 \pm 0.4 \mathrm{c}$ \\
\hline
\end{tabular}

Table 1: Effect on the time to petal senescence of phytosphingosine together with $\mathrm{LaCl} 3$, an inhibitor of $\mathrm{Ca} 2+$ effects, 1Data are means of ten replicate flowers, Data with a different letter are significantly different $(\mathrm{P} \geq 0.05)$

\section{Myriocin}

The initial biosynthetic step of sphingolipid biosynthesis is catalyzed by serine palmitoyltrans-ferase (SPT, Figure 1), which is inhibited by myriocin (Chen et al. 1999). Myriocin significantly $(P=0.05)$ hastened the visible PCD symptoms when a concentration of $0.3 \mu \mathrm{M}$ was included in the aqueous solution used for standing isolated Petunia flowers (Figure 4A). At higher concentrations, it increasingly further hastened these PCD symptoms. The highest concentration tested was $10 \mu \mathrm{M}$. It reduced the time to the visible PCD symptoms to less than half (Figure 4A). In these experiments myriocin was first dissolved in DMSO. DMSO controls had no effect (not shown). In other tests myriocin was first dissolved in potassium hydroxide. Although the solvent had no effect on the time to the visible symptoms, myriocin was much less effective. In these experiments it was only statistically shortening the time to the symptoms at $5 \mu \mathrm{M}$, and at $10 \mu \mathrm{M}$ it had an effect similar to that of $0.6 \mu \mathrm{M}$ in the experiment in which it was first dissolved in DMSO.

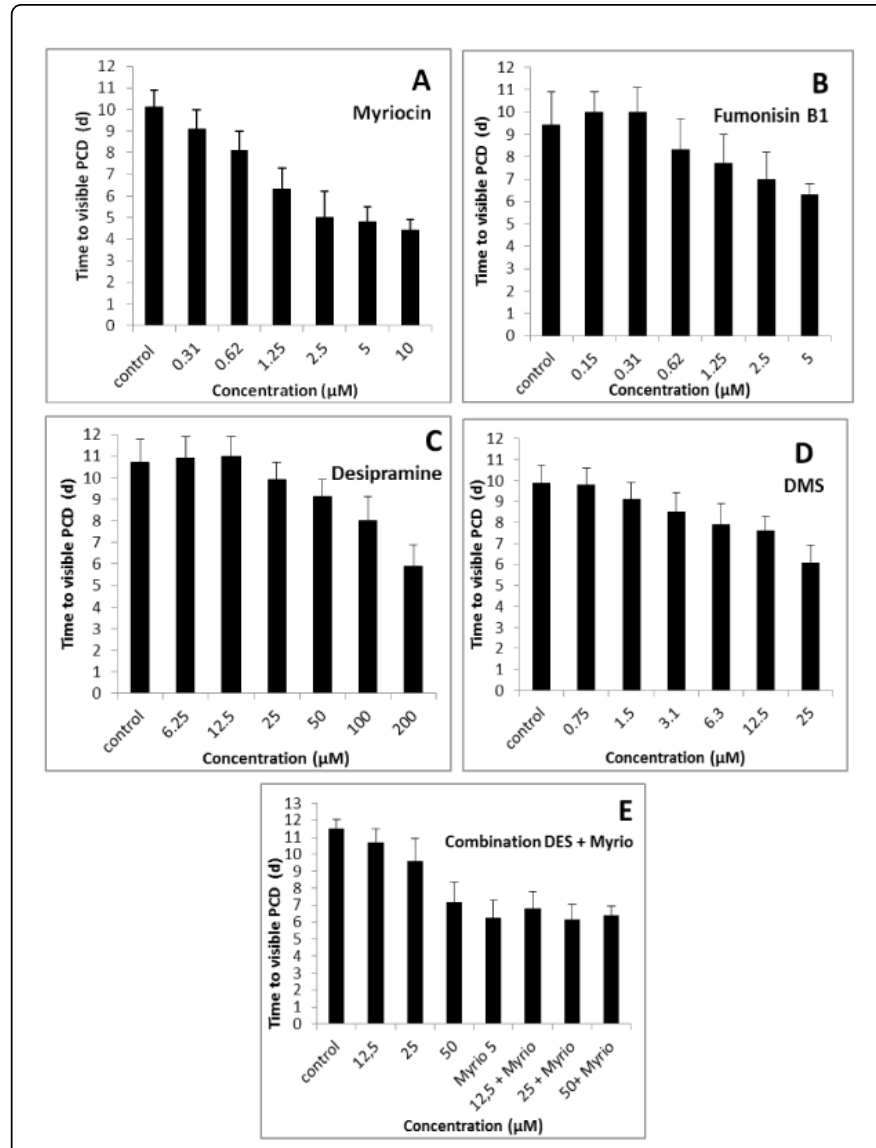

Figure 4: Effect of continuous treatment with inhibitors of sphingolipid biosynthesis on the time to visible PCD in petals of isolated Petunia flowers placed with the pedicel in aqueous solution. (A) myriocin (B) fumonisin B1 (C) desipramine, (D) DMS (N,Ndimethylsphingosine), (E) Combined treatment with myriocin and desipramine.. In these experiments DMS and myriocin were first dissolved in $10 \mathrm{ml}$ pure DMSO. Desipramine and fumonisin B1 were directly dissolved in water. No effect was found of DMSO control.

\section{DMS (N,N-dimethylsphingosine)}

DMS decreases the activity of sphingolipid kinases such as sphinganine kinase, phytosphingosine kinase, ceramide kinase and spingosine kinase. These enzymes phosphorylate their substrates (Figure 1). DMS significantly $(\mathrm{P}=0.05)$ hastened the visible PCD symptoms at a concentration of $1.5 \mu \mathrm{M}$ in the aqueous solution (Figure $4 \mathrm{~B})$. The effect became larger at higher concentrations. The highest concentration tested was $25 \mu \mathrm{M}$ (Figure 4D). DMS was first dissolved in DMSO. A control for this solvent showed no effect on the parameter studied.

\section{Fumonisin B1}

Fumonisin B1 is an inhibitor of sphinganine- $\mathrm{N}$-acyltransferase, which catalyses the conversion of sphinganine to dihydroxyceramide (Figure 1) [14]. When using myriocin, DMS, or desipramine, the visible symptoms at the time of visible wilting were the same as in the 
untreated controls: bluing of the tepals rapidly followed by complete turgor loss. These symptoms were also found in the experiments using fumonisin B1, but an additional symptom was observed. The petal surface showed blue discoloration which was concentrated near the center of the petals. The symptoms were localized in the epidermal cells which also showed local collapse (data not shown). Fumonisin B1 hastened the time to petal wilting from a concentration of $0.6 \mu \mathrm{M}$ (Figure $4 \mathrm{~B}$ ). At $5 \mu \mathrm{M}$ it hastened the symptoms to more than half of the time of that in the controls (Figure $4 \mathrm{~B}$ ).

\section{Desipramine}

The conversion of ceramide to sphingosine is catalysed by ceramidases. Plants contain both neutral and acid ceramidase. Desipramine is an inhibitor of acid ceramidase [8]. Desipramine hastened the visible senescence symptoms from a concentration of 25 $\mu \mathrm{M}$ (Figure $4 \mathrm{C}$ ). At $100 \mu \mathrm{M}$ it hastened the symptoms to more than half of the time of that in the controls (Figure 4C). Desipramine was also applied together with myriocin. The combination of inhibitors had a larger effect than myriocin alone (Figure $4 \mathrm{E}$ ).

\section{Discussion}

\section{Effects of exogenous sphingolipids}

We observed a hastening of PCD when feeding Petunia petals with some main plant sphingolipids. This suggests that maintenance of low sphingolipid levels in the cell is important for survival. The largest effects, at similar exogenous concentrations, were observed with sphinganine and phytosphingosine. Similar effects on PCD of feeding of plants with sphingolipids have been reported with ceramide [12,13], sphinganine, phytosphingosine [11] and sphingosine [8]. Effects of dihydroceramide in plants have apparently not been reported before. Islam et al. in 2012 found that sphingosine was absent in Arabidopsis (Brassicaceae), Glycine max (Fabaceae) and Pyrus malus (Rosaceae), but present in all Poales investigated, as well as in potato and tomato (Solanaceae). This suggests that it might also occur in the solanaceous Petunia.

\section{Role of ethylene, ROS, and Ca2+}

We tested if the applied sphingolipids might act through ethylene. PCD in many flowers, including Petunia, is due to an autocatalytic rise in ethylene production [20,27]. Autocatalysis depends on perception of the increase in ethylene, which then further increases ethylene synthesis. This explains why PCD in these flowers is delayed by blocking the ethylene receptors by silver thiosulphate (STS), an anion that is mobile in plants [28]. We observed that STS counteracted the effect of phytosphingosin. This suggests that phytosphingosin acted on PCD through ethylene. An increase in ethylene production occurs as a result of any type of stress. High levels of phytophingosine in the cells might induce such a stress. The data do not exclude that sphingolipids accumulate in the vacuolar membrane, but they render this idea less likely. The rupture of the vacuolar membrane is the end product of ethylene signaling. Phytosphingosine is apparently upstream of ethylene signaling rather than downstream.

Some reports suggested that the sphingolipids induce the production of ROS, such as hydrogen peroxide, which is toxic to cells or can act as a second messenger. We tested if the effect of phytospingosine would be alleviated by the further inclusion of $\mathrm{N}$ acetyl-L-cysteine (Nac). Nac is a scavenger of ROS. If phytosphingosine acted through ROS, we expected Nac to alleviate its effect. No such effect of Nac was observed. However, Nac was toxic by itself. We therefore also tested feeding of phytosphingosine together with ascorbic acid, another antioxidant (backslash) ROS scavenger. Ascorbic acid slightly increased the time to senescence in the controls and had about the same effects together with phytophingosine. The effect of sphytophingosine was not abrogated. These data suggest that phytophingosine did not act through ROS.

$\mathrm{Ca} 2+$ is a signal transduction molecule that results in specific stimulation of gene expression. $\mathrm{Ca} 2+$ transport through plant membranes is mediated by ion channels. These channels are inhibited by $\mathrm{La} 3+[29-31]$. In our tests, $\mathrm{La} 3+$ did not alleviate the effect of phytosphingosine. However, La3+ by itself hastened PCD. The data therefore neither contradict nor provide support for the hypothesis that $\mathrm{Ca} 2+$ is involved as a messenger in phytosphingosine-induced hastening of PCD.

\section{Effects of inhibitors of sphingolipid biosynthesis}

In order to obtain further insight into the role of endogenous sphingolipid levels, we applied sphingolipid biosynthesis inbibitors. All inhibitors, when tested individually, advanced the time to PCD in Petunia petals. The inhibitors are expected to result in accumulation of at least their substrate and in a decrease in the concentration of at least their direct product (Figure 1).

Desipramine, therefore, would be expected to decrease levels of sphingosine and sphingosine1-phosphate, and to increase ceramide concentrations. Feeding of animal cell lines with desipramine indeed resulted in ceramide accumulation, from a desipramine concentration of $5 \mu \mathrm{M}$ [32]. Desipramine also inhibits the conversion of sphingosines to sphingomyelins (catalysed by sphingomyelin synthase), a reaction found in animals, not in plants [16].

The present data on desipramine seem in contrast to similar experiments in animal cells, where sphingosine induced PCD while ceramide did not. Using a siRNA approach Ullio et al. in 2012 reported that blocking acid ceramidase activity abrogated induced lysosomal permeabilisation and cell death in animal cells. These data suggested that death was due to sphingosine and that ceramide had no effect. The present data, by contrast, indicated that endogenous ceramide induced earlyt PCD in the present plant system, just as exogenous ceramide did.

Fumonisin B1 is expected to increase the concentrations of phytosphingosine and sphinganine, and their respective 1-phosphates, and to decrease the concentration of dihydroceramide and ceramide (Figure

1). Treatment of corn shoots with $1 \mu \mathrm{M}$ fumonisin $\mathrm{B} 1$ resulted in 8-10 fold increase in sphinganine and phytosphingosine levels. The toxicity of fumonisin was thought to be due mainly to phytosphingosine accumulation [11]. Treatment with fumonisin B1 in maize also resulted in higher levels of sphinganine-1-phosphate and phytosphinganin-1-phosphate [33]. Arabidopis has three ceramide synthase homologs. Each isoform showed a characteristic preference regarding fatty acid chain length as well as sphingoid base hydroxylation. Plants in which one of the three ceramide synthase homologs was knocked out showed spontaneous PCD, after extended culture under short-day conditions. The data suggested that the spontaneous PCD was due either to the accumulation of free trihydroxy sphingoid bases and/or that of ceramide species with $\mathrm{C} 16$ fatty acid. These data indicate that the effect of fumonisin B1 on PCD 
might be more complex than simply due to accumulation of sphinganine and phytosphingosine. A role of a decrease in (dihydro)ceramide can also not be excluded.

DMS inhibits the formation of sphingolipid-1-phosphates. Such products exhibit anti-PCD activity. For example, PCD was induced by ceramide, but its effect was partially blocked by the simultaneous gift of ceramide-1-phosphate [12]. Similarly, feeding with sphinganine induced PCD which was specifically counteracted by sphinganine-1phosphate [8]. The effect of DMS can be explained, apparently, by the increase in substrate and decrease in product. The effect of DMS is in accordance with the effect of feeding with the main plant sphingolipids.

Myriocin is expected to decrease the levels of sphingolipids, at least of 3- ketosphin- ganine, sphinganine,and 4-hydroxysphinganine. Feeding with a combination of myriocin and desipramine (which converts ceramide to sphingosine) hastened PCD more than feeding with myriocin alone. This suggests that myriocin did not prevent the synthesis of sphingolipids upstream of the biosynthesis pathway.

\section{Possible mechanism of the effect of sphingolipids on PCD}

Our data suggest that an increase in endogenous concentrations of non-phosphorylated sphingolipids induces early PCD. The mechanism of action is not known. We hypothesized that spingolipids might disrupt the vacuolar membrane, thereby inducing membrane rupture, as has been suggested for animal cells. This hypothesis is not likely, as the effect of at least one sphingolipid (phytosphingosine) was abrogated by blocking ethylene perception and synthesis. This suggest that the sphingolipid acted upstream of ethylene, possibly by perturbing metabolism, which induced an increase in ethylene synthesis.

\section{References}

1. Obara, K., Kuriyama, H., Fukuda H. 2001. Direct evidence of active and rapid nuclear degradation triggered by vacuole rupture during programmed cell death in zinnia. Plant Physiology 125, 615-626.

2. Jones AM (2001) Programmed cell death in development and defense. Plant Physiol 125: 94-97.

3. Evans, D.E. 2003. Aerenchyma formation. New Phytologist 161, 35-49.

4. Boya P, Kroemer G (2008) Lysosomal membrane permeabilization in cell death. Oncogene 27: 6434-6451.

5. Kirkegaard T, Jäättelä M (2009) Lysosomal involvement in cell death and cancer. Biochim Biophys Acta 1793: 746-754.

6. Ullio C, Casas J, Brunk UT, Sala G, Fabriàs G, Ghidoni R, Bonelli G, Baccino FM, Autelli R. 2012. Sphingosine mediates TNF?-induced lysosomal membrane permeabilization and ensuing programmed cell death in hepatoma cells. The Journal of Lipid Research 53, 1134-1143.

7. Hannun YA, Obeid LM (2008) Principles of bioactive lipid signalling: lessons from sphingolipids. Nat Rev Mol Cell Biol 9: 139-150.

8. Shi L, Bielawski J, Mu J, Dong H, Teng C, et al. (2007) Involvement of sphingoid bases in mediating reactive oxygen intermediate production and programmed cell death in Arabidopsis. Cell Res 17: 1030-1040.

9. Pewzner-Jung Y, Ben-Dor S, Futerman AH (2006) When do Lasses (longevity assurance genes) become CerS (ceramide synthases)?: Insights into the regulation of ceramide synthesis. J Biol Chem 281: 25001-25005.

10. Berkey R, Bendigeri D, Xiao S (2012) Sphingolipids and plant defense/ disease: the "death" connection and beyond. Front Plant Sci 3: 68

11. Wright BS, Snow JW, O'Brien TC, Lynch DV (2003) Synthesis of 4hydroxysphinganine and characterization of sphinganine hydroxylase activity in corn. Arch Biochem Biophys 415: 184-192.
12. Liang H, Yao N, Song JT, Luo S, Lu H, et al. (2003) Ceramides modulate programmed cell death in plants. Genes Dev 17: 2636-2641.

13. Peters J, Chin CK (2007) Potassium loss is involved in tobacco cell death induced by palmitoleic acid and ceramide. Arch Biochem Biophys 465: 180-186.।

14. Wang H, Li J, Bostock RM, Gilchrist DG (1996) Apoptosis: A Functional Paradigm for Programmed Plant Cell Death Induced by a Host-Selective Phytotoxin and Invoked during Development. Plant Cell 8: 375-391.

15. Spassieva SD, Markham JE, Hille J (2002) The plant disease resistance gene Asc-1 prevents disruption of sphingolipid metabolism during AALtoxin-induced programmed cell death. Plant J 32: 561-572.

16. Lynch DV, Dunn TM. 2004.[HTML] An introduction to plant sphingolipids and a review of recent advances in understanding their metabolism and function. New Phytologist 161, 677-702.

17. Ternes P, Feussner K, Werner S, Lerche J, Iven T, et al. (2011) Disruption of the ceramide synthase LOH1 causes spontaneous cell death in Arabidopsis thaliana. New Phytol 192: 841-854.

18. Bartke N, Fischbeck A, Humpf HU (2006) Analysis of sphingolipids in potatoes (Solanum tuberosum L.) and sweet potatoes (Ipomoea batatas (L.) Lam.) by reversed phase high-performance liquid chromatography electrospray ionization tandem mass spectrometry (HPLC-ESI-MS/MS). Mol Nutr Food Res 50: 1201-1211.

19. Valsecchi, M., L. Mauri, R. Casellato, M.G. Ciampa, L. Rizza, A. Bonina, F. Bonina, and S. Sonnino. (2012). Ceramides as possible nutraceutical compounds: characterization of the ceramides of the Moro blood orange (Citrus sinensis). Journal of Agricultural Food Chemistry 60, 10103-10110.

20. Langston BJ, Bai S, Jones ML (2005) Increases in DNA fragmentation and induction of a senescence-specific nuclease are delayed during corolla senescence in ethylene-insensitive (etr1-1) transgenic petunias. J Exp Bot 56: $15-23$.

21. van Doorn WG (2011) Classes of programmed cell death in plants, compared to those in animals. J Exp Bot 62: 4749-4761.

22. Shaw JF, Chen HH, Tsai MF, Kuo CI, Huang LC. 2002. Extended flower longevity of Petunia hybrida plants transformed with boers, a mutated ERS gene of Brassica oleracea. Molecular Breeding 9, 211-216.

23. Yoshida K, Toyama-Kato Y, Kameda K, Kondo T (2003) Sepal color variation of Hydrangea macrophylla and vacuolar $\mathrm{pH}$ measured with a proton-selective microelectrode. Plant Cell Physiol 44: 262-268.

24. Quattrocchio F, Verweij W, Kroon A, Spelt C, Mol J, et al. (2006) PH4 of Petunia is an R2R3 MYB protein that activates vacuolar acidification through interactions with basic-helix-loop-helix transcription factors of the anthocyanin pathway. Plant Cell 18: 1274-1291.

25. Koes RE, Van Blokland R, Quattrocchio F, Van Tunen AJ, Mol J (1990) Chalcone Synthase Promoters in Petunia Are Active in Pigmented and Unpigmented Cell Types. Plant Cell 2: 379-392.

26. Causeret C, Geeraert L, Van der Hoeven G, Mannaerts GP, Van Veldhoven PP (2000) Further characterization of rat dihydroceramide desaturase: tissue distribution, subcellular localization, and substrate specificity. Lipids 35: 1117-1125.

27. van Doorn WG, Woltering EJ (2008) Physiology and molecular biology of petal senescence. J Exp Bot 59: 453-480.

28. Veen H, van de Geijn SC (1978) Mobility and ionic form of silver as related to longevity of cut carnations. Planta 140: 93-96.

29. Demidchik V, Maathuis FJ (2007) Physiological roles of nonselective cation channels in plants: from salt stress to signalling and development. New Phytol 175: 387-404.

30. Demidchik V, Bowen HC, Maathuis FJ, Shabala SN, Tester MA, et al. (2002) Arabidopsis thaliana root non-selective cation channels mediate calcium uptake and are involved in growth. Plant J 32: 799-808.

31. Kurusu T, Kuchitsu K, Nakano M, Nakayama Y, Iida H (2013) Plant mechanosensing and Ca2+ transport. Trends Plant Sci 18: 227-233.

32. Elojeimy S, Holman DH, Liu X, El-Zawahry A, Villani M, et al. (2006) New insights on the use of desipramine as an inhibitor for acid ceramidase. FEBS Lett 580: 4751-4756. 
Citation: Prisa D, Burchi G (2015) Possible Role of Sphingolipids in Developmental Programmed Cell Death of Petunia Petals. Biol syst Open Access 4: 146. doi:10.4172/2329-6577.1000146

Page 7 of 7

33. Williams LD, Glenn AE, Zimeri AM, Bacon CW, Smith MA, et al. (2007) Fumonisin disruption of ceramide biosynthesis in maize roots and the effects on plant development and Fusarium verticillioides-induced seedling disease. J Agric Food Chem 55: 2937-2946. 